\title{
In Situ Scanning Transmission Electron Microscopy Observations of Fracture at the Atomic Scale
}

\author{
Lingli Huang $\odot,{ }^{1, *}$ Fangyuan Zheng, ${ }^{2, *}$ Qingming Deng $\odot,{ }^{3, *}$ Quoc Huy Thi $\odot,{ }^{1, *}$ Lok Wing Wong $\odot,{ }^{2}$ Yuan Cai, ${ }^{4}$ \\ Ning Wang, ${ }^{4}$ Chun-Sing Lee, ${ }^{1}$ Shu Ping Lau, ${ }^{2}$ Manish Chhowalla, ${ }^{5} \mathrm{Ju} \mathrm{Li},{ }^{6}$ Thuc Hue Ly๑ ${ }^{1,7, \dagger}$ and Jiong Zhao $\odot^{2,8, \sharp}$ \\ ${ }^{1}$ Department of Chemistry and Center of Super-Diamond \& Advanced Films (COSDAF), City University of Hong Kong, \\ Kowloon, Hong Kong, China \\ ${ }^{2}$ Department of Applied Physics, The Hong Kong Polytechnic University, Kowloon, Hong Kong, China \\ ${ }^{3}$ Physics department and Jiangsu Key Laboratory for Chemistry of Low-Dimensional Materials, Huaiyin Normal University, \\ Huaian 223300, China \\ ${ }^{4}$ Department of Physics, Hong Kong University of Science and Technology, Clear water bay, Hong Kong, China \\ ${ }^{5}$ Department of Materials Science and Metallurgy, University of Cambridge, Cambridge, United Kingdom \\ ${ }^{6}$ Department of Nuclear Science and Engineering and Department of Materials Science and Engineering, \\ Massachusetts Institute of Technology, Cambridge, Massachusetts 02139, USA \\ ${ }^{7}$ City University of Hong Kong Shenzhen Research Institute, Shenzhen, China \\ ${ }^{8}$ The Hong Kong Polytechnic University Shenzhen Research Institute, Shenzhen, China
}

(Received 16 June 2020; revised 10 September 2020; accepted 16 October 2020; published 9 December 2020)

\begin{abstract}
The formation, propagation, and structure of nanoscale cracks determine the failure mechanics of engineered materials. Herein, we have captured, with atomic resolution and in real time, unit cell-by-unit cell lattice-trapped cracking in two-dimensional (2D) rhenium disulfide $\left(\mathrm{ReS}_{2}\right)$ using in situ aberration corrected scanning transmission electron microscopy (STEM). Our real time observations of atomic configurations and corresponding strain fields in propagating cracks directly reveal the atomistic fracture mechanisms. The entirely brittle fracture with non-blunted crack tips as well as perfect healing of cracks have been observed. The mode I fracture toughness of $2 \mathrm{D} \mathrm{ReS}$ is measured. Our experiments have bridged the linear elastic deformation zone and the ultimate nm-sized nonlinear deformation zone inside the crack tip. The dynamics of fracture has been explained by the atomic lattice trapping model. The direct visualization on the strain field in the ongoing crack tips and the gained insights of discrete bond breaking or healing in cracks will facilitate deeper insights into how atoms are able to withstand exceptionally large strains at the crack tips.
\end{abstract}

DOI: 10.1103/PhysRevLett.125.246102

The fundamental limitation in designing fracture resistant materials using elastic stress analysis is the presence of cracks that increase the local stresses near crack tipscausing real components to fail at much lower stresses than in ideal specimens. Despite decades of study, atomic structures of propagating crack tips (when loaded close to or over the Griffith load) in crystals have not been experimentally observed, and atomic resolution imaging of the crack tip and its propagation have remained elusive [1]. This has led to notable discrepancies between theoretical models and experiments [2-8]. While some controlled (referred to as stopped) cracks have been imaged in transmission electron microscopy (TEM) [9,10], and some mesoscopic fracture tests $[11,12]$ and high resolution TEM observations $[13,14]$

Published by the American Physical Society under the terms of the Creative Commons Attribution 4.0 International license. Further distribution of this work must maintain attribution to the author(s) and the published article's title, journal citation, and DOI. on the atomically blunted crack tip zones were reported recently, the atomic structure of crack tips-whether they are atomically sharp or blunt [15-18]—in brittle materials remains unresolved. In particular, electron beam damage was present and the stress field in the crack tip zone has been released in previous TEM studies $[13,14]$, which preclude further analysis on the intrinsic mechanical properties.

On the other hand, while theoretical calculations describe far field stress in front of or behind the crack tips, they are less successful in describing the divergent stress field near the crack tip because the atomic cohesive forces are well beyond the linear elastic regime but still provide bonding across the crack faces. Atomistic simulations of crack tips [5-8,19,20] require details of interatomic forces, which could be obtained experimentally. Regarding crack dynamics, a number of atomic-scale theories [21-23] have been established, however, without experimental verifications at the atomic scale. Many macroscopic experiments have revealed large discrepancies between the cracks in real brittle materials and the dynamical fracture theories, suggesting there are still unknown mechanisms [24]. 
The central difficulty in experimental real time capture of atomic structure of a fast propagating crack is the tradeoff between spatial and temporal resolution, as well as the control of the electron beam effect. Here we show that cracks in 2D materials [25] provide the ideal platform for studying fundamentals of cracks with atomic resolutions using in situ STEM. The ultrahigh flexibility and lattice switching capability of $2 \mathrm{D} \mathrm{ReS} \mathrm{S}_{2}$ membranes allows for the angstrom-scale displacement control of the vicinity of ongoing crack tip zone. Moreover, using the beam scanning mode, the cracking events were able to be analyzed either without or with the beam effect.

Anisotropic monolayered 2D $\operatorname{ReS}_{2}$ [26] [Fig. 1(a)] was used to understand the structure of the crack tip and its propagation behavior at the atomic scale. The 2D samples were grown by the chemical vapor deposition (CVD) method (Supplemental Material [27], Fig. S1), and prior to the in situ STEM experiments, the defectless nature of our specimens have been first confirmed by the atomic force microscopy (AFM) indentation tests, the nearly ideal strengths have been demonstrated (Supplemental Material [27], Figs. S2-S4). Further, the $2 \mathrm{D} \mathrm{ReS}_{2}$ samples were transferred into Cs-corrected TEM (JEOL, ARM 200F) working under $60 \mathrm{kV}$ at room temperature. Intensive electron beam irradiation in TEM mode was initially used to generate large cracks on the sample (Supplemental Material [27]), which naturally led to several subcracks extending outside of the beam irradiation area [Fig. 1(b)], hence the these subcrack positions for in situ observations did not suffer from the intensive beam irradiation in the beginning. The following in situ STEM imaging condition was carefully controlled to avoid knockout damage on the sample (Fig. S5 [27]).

We then focused on the further cracking processes using STEM. The mode I crack-which occurs when tensile load is applied perpendicular to the crack direction-was most commonly observed. $\mathrm{ReS}_{2}$ crystal is able to switch the crystal lattice directions (between $\boldsymbol{a}$ and $\boldsymbol{b}$ ) under shear strain. Noted that the lattices of $\mathrm{ReS}_{2}$ were switched in the postcrack edges [Fig. 1(c)], there is one tensile stress enhanced zone close to the crack tip zone due to the lattice expansion by the switched areas [inset of Fig. 1(c)], and it led to a tensile opening stress for mode I fracture on the crack tip zone [Fig. 1(c) and Fig. S6 [27] ]. Two snapshots of such a mode I crack tip along the $\boldsymbol{a}$ axis of $\mathrm{ReS}_{2}$, acquired through high angle annular dark field (HAADF) imaging are shown in Fig. 1(d). Wiener filtering was applied on HAADF images for reduction of noises. The (a)

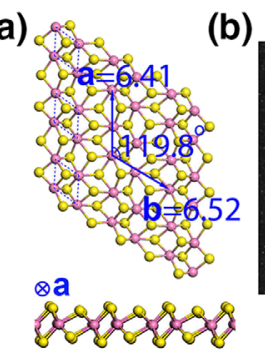

(g)

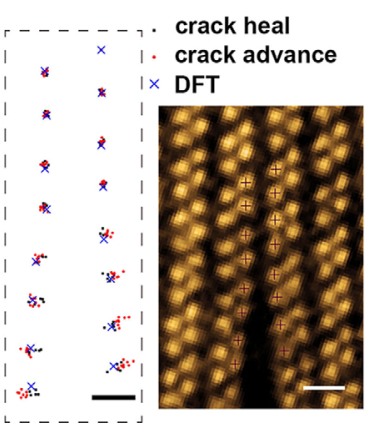

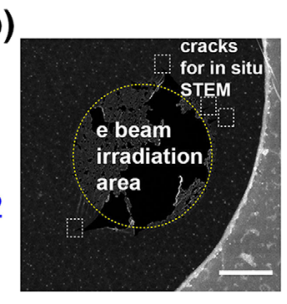

(f)

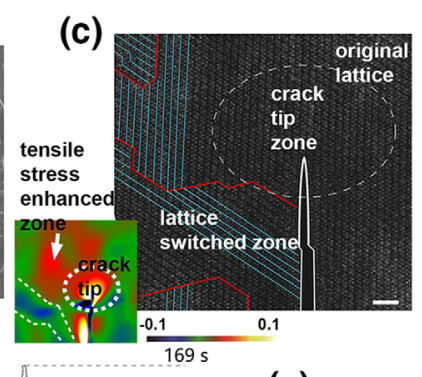

(e) (d)

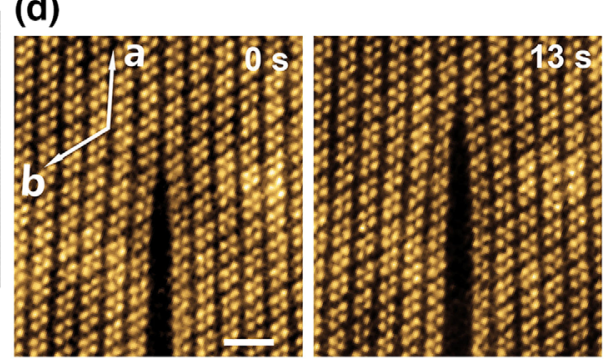

healed by 1 unit cell

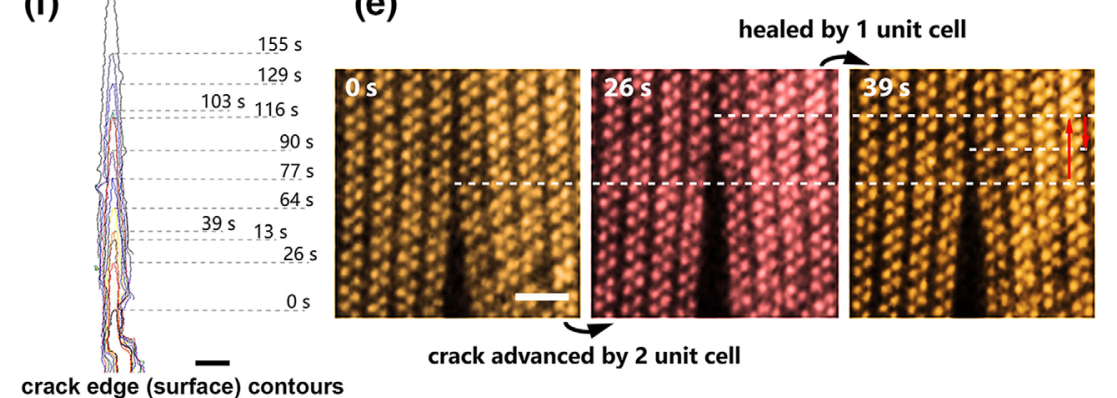

crack edge (surface) contours

FIG. 1. The mode I cracks in $2 \mathrm{D} \operatorname{ReS}_{2}$ along the $\boldsymbol{a}$ axis. (a) The monoclinic crystal structure model of $2 \mathrm{D}$ monolayer ReS 2 , plan view (upper) and side view (lower), with $\boldsymbol{a}, \boldsymbol{b}$ as basis vectors. (b) The generation of initial cracks by $e$ beam irradiation. Scale bar $=100 \mathrm{~nm}$. (c) HAADF showing the mode I crack driven by postedge lattice switching. Scale bar $=2 \mathrm{~nm}$. Inset shows the GPA strain analysis of this area for normal stress perpendicular to the crack direction. (d) Two HAADF snapshots of the crack tip zone of an ongoing crack. Crystal directions are highlighted by arrows. These directions apply to all of the HAADF images in Fig. 1. All images have been drift compensated and aligned to show the same positions of the specimen. False colors are applied on HAADF images. Brighter spots represent positions of Re atoms, while $\mathrm{S}$ atoms are barely visible. Scale bar $=1 \mathrm{~nm}$. (e) In situ snapshot series of cracking and healing processes in $2 \mathrm{D} \mathrm{ReS}_{2}$ by single steps (unit cell). Cracking in yellow and healing in red. Scale bar $=1 \mathrm{~nm}$. (f) Evolution of a crack edge contour observed by in situ STEM with observation times marked, scale bar $=2 \mathrm{~nm}$. (g) The inner most 16 Re atomic positions inside the crack tips extracted from 13 experimental images (black: crack heal; red: crack advance), an example HAADF image shown in the right side, and the DFT simulation results are shown as blue crosses. All sets of atomic position data are aligned using the upper right Re atom's position as reference. Scale bar $=0.2 \mathrm{~nm}$. 
crack was straight and atomically smooth. We find that continuous cracking is discretized down to the atomic scale. That is, cracking is periodic and repeatable along the unit cells. Further observations suggest that both crack advance and crack healing can occur [Fig. 1(e)]. The continuous propagation of one crack tip (outline edge contours) in our experiments is depicted in Fig. 1(f).

Although fracture is usually irreversible, we were able to observe crack healing by rebonding of free edges behind the crack front [26 to $39 \mathrm{~s}$ in Fig. 1(e)]. The maximum length of continuous crack healing extends three unit cells in our observations (Fig. S7 [27]). The healing implies that the fracture is entirely brittle, and plasticity or reconstructions are absent in the $\operatorname{ReS}_{2}$. The inner most Re atom positions within the crack tip zones were extracted from experimental images and overlaid with our density functional theory (DFT) analysis [28] results [Fig. 1(g)]. The experimental atomic positions for crack advance and healing were distinctly separated, in agreement with the
DFT simulated atomic structures. Our DFT analysis results [Fig. 2(a) and Fig. S8 [27]] on mode I cracks along the $\boldsymbol{a}$ axis in $\mathrm{ReS}_{2}$ also reproduce the atomically smooth edges and the sequential rupture and healing of Re-S bonding at the crack tips, depending on the initial strain applied. The free edges formed due to cracking were occupied by dangling $\mathrm{S}$ atoms, suggesting that there was neither knockout of atoms by electron beam nor loss of atom during fracture process [Fig. 2(b) and Fig. S9 [27] ]. The sequential in situ STEM observations sometimes captured the discontinuous lines in the images [Fig. 2(c)], which we will discuss in detail in the dynamic analysis below.

The strain fields near tip zone were mapped (Fig. S10 [27]) through the geometric phase analysis (GPA) [29] on the HAADF images. Meanwhile, utilizing the experimentally determined atomic structure of crack tips, the atomic strains were quantitatively analyzed for each half unit cell or Voronoi cell [30] to study the critical condition for crack advance (growth) or healing. In Fig. 2(d), crack heal (a)

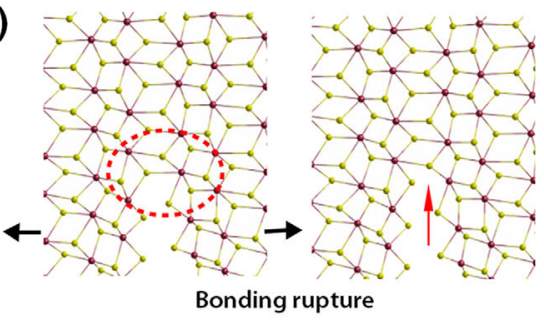

(b)

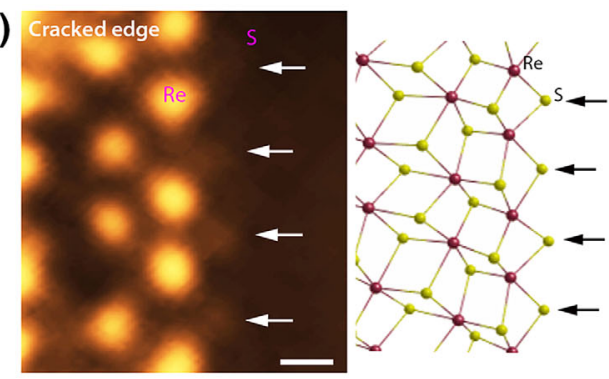

(c)

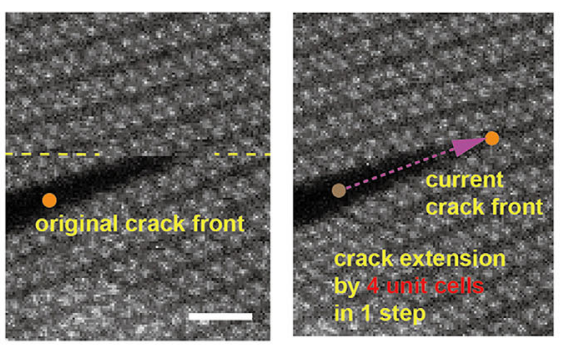

(d) crack heal
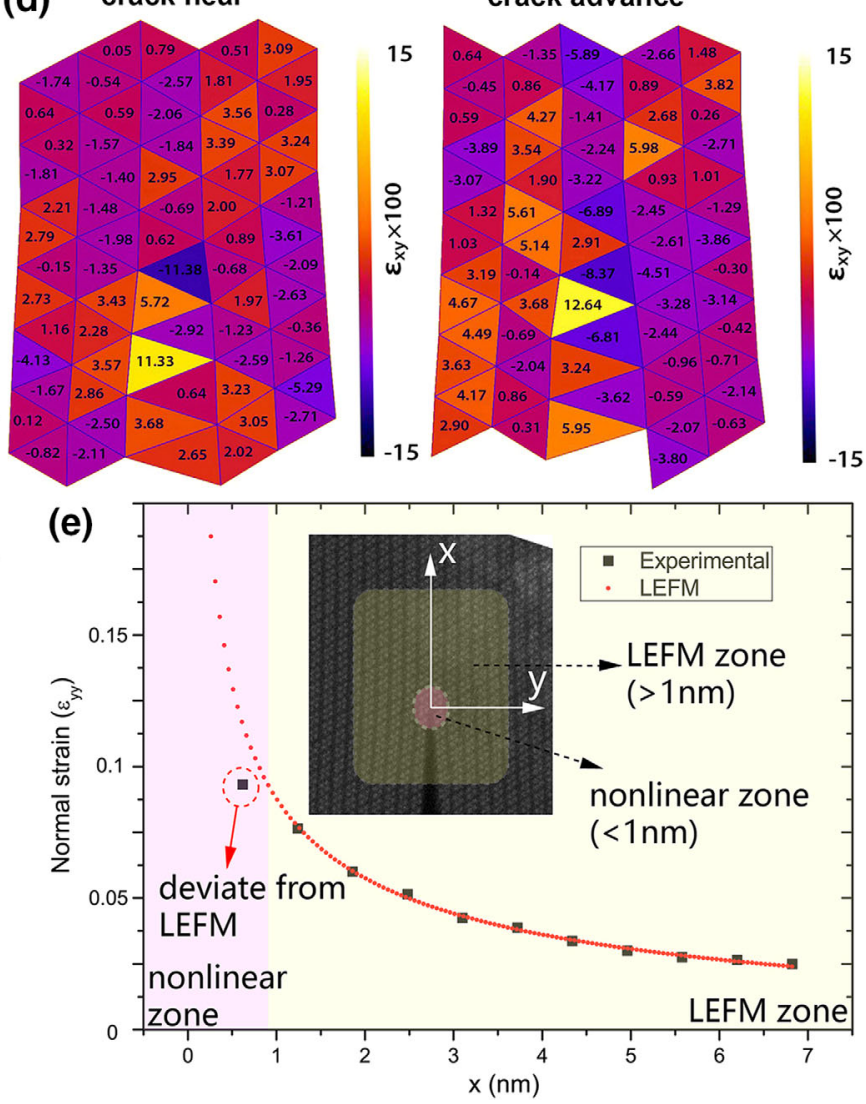

FIG. 2. Atomic-scale strain analysis on the crack tip zones. (a) Two sequential snapshots of DFT simulated mode I cracks in $\mathrm{ReS}_{2}$. The ruptured Re-S bond is marked by red arrow. (b) Magnified HAADF image of one $\mathrm{ReS}_{2}$ cracked edge, white arrows indicate the position of dangling $\mathrm{S}$ atoms. Scale bar $=0.3 \mathrm{~nm}$. Corresponding DFT results of $\mathrm{ReS}_{2}$ cracked edge shown on right side. (c) The discontinuity in STEM image caused by crack move when the electron beam is scanning closely to the crack tip. Two consecutive STEM snapshots of the same position in monolayer $\operatorname{ReS}_{2}$ sample showing the crack instantly move by 4 unit cells when the beam scanning reaches the yellow dashed line position. Scale bar $=1 \mathrm{~nm}$. (d) Shear strain results (color encoded, discretized by half unit cell) of $\mathrm{ReS}_{2} \mathrm{Mode} \mathrm{I}$ crack and healing along the $\boldsymbol{a}$ axis in our experiments. (e) The normal strain on the $x$ axis (shown in the inset) for mode I crack in $1 L$ $\mathrm{ReS}_{2}$. The strain inside the $1 \mathrm{~nm}$ region deviate from the LEFM theory. 
(a)

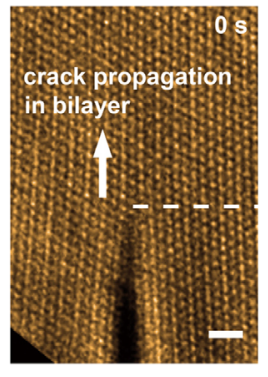

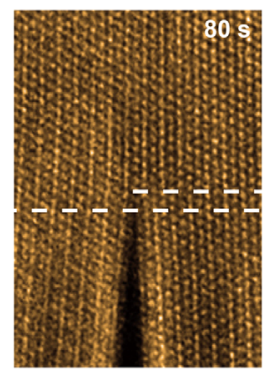

(b)

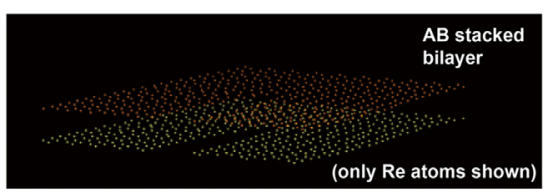

(c)

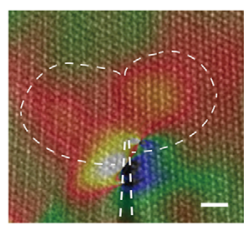

(d)

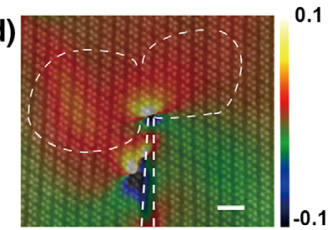

FIG. 3. Cracks in bilayers. (a) The STEM snapshots of a crack in bilayer $(2 L) \operatorname{ReS}_{2}$ which propagate by one unit cell. Scale bar $=1 \mathrm{~nm}$. (b) Scheme for the bilayer $\mathrm{ReS}_{2}$ and the always synchronized cracking in the upper and lower layers. (c) The normal (tensile) strain $\left(e_{y y}\right)$ distribution corresponding to (a). Scale bar $=1 \mathrm{~nm}$. (d) The normal strain distribution $\left(e_{y y}\right)$ for another $1 L \operatorname{ReS}_{2}$ sample, showing similar strain fields for $1 L$ and $2 L$ specimens. Scale bar $=1 \mathrm{~nm}$.

and crack advance are two example snapshots taken in our experiment and here we show the lattice discrete strain analysis results on these STEM snapshots. The lattice discrete strain is calculated by the atomic displacement directly measured from STEM images. The formulation of the lattice discrete strain analysis (Fig. S11 [27]) and detailed analysis methods are introduced in the Supplemental Material [27]. According to the obtained atomic strains, in the range of $1-5 \mathrm{~nm}$ away from the crack tips, the asymptotic strain field by the linear elastic fracture mechanics (LEFM) [31,32] [Fig. 2(e) and Fig. S12 [27]] and the $J$ integral approach [33] (Fig. S13 [27]) still hold, showing the fracture process induced a well-defined mode I crack. For the area out of the $1 \mathrm{~nm}$ tip zone, the LEFM relationship $\varepsilon_{y y} E=$ $K(2 \pi x)^{-1 / 2}$ can still be used to describe our experimental results [Fig. 2(e)], $E$ is modulus. The stress intensity factor $\left(K_{I}\right)$ is derived from the fitting parameter in the LEFM zone. For the area within the $1 \mathrm{~nm}$ tip zone, the strain field deviates from the LEFM and the $J$ integral theory, while nonlinear deformation dominates.

We carried out similar experiments on bilayer $\mathrm{ReS}_{2}$. For the commensurately stacked bilayer $\operatorname{ReS}_{2}$ samples, the in situ TEM observed cracking in both layers are synchronized, which yield similar atomically sharp tips as monolayer $\mathrm{ReS}_{2}$ [Figs. 3(a), 3(b)], the strain distributions are almost identical for the crack tips in bilayers and monolayers [Figs. 3(c), 3(d)]. In contrast, the incommensurately stacked bilayer $\operatorname{ReS}_{2}$ could have roughened crack surfaces and different crack paths or directions in the two layers (Fig. S14, S15 [27]). Therefore, our cracking experiments directly exhibit the effect of interlayer vdW interactions. With stronger interactions (commensurate stacking), the basal plane strains can be transmitted through different layers, while under weak interaction (incommensurate stacking) the basal plane strains will have less correlation.

The macroscopic fracture criterion has been well established in the classical fracture mechanics [31,32]. However, in reality the loading required for fracture needs to exceed the Griffith load (when the strain energy equals to the new surface energy). Here our experiments have confirmed the LEFM can be applied until very small regions (nm sized) within the crack tip for entirely brittle materials, and the stress intensity factor $(K)$ can be obtained by fitting the strain distributions with LEFM theory [Fig. 2(e), and Fig. S12 [27] ]. The statistics on all the in situ observations on the mode I cracks give the result shown in Fig. 4(a). Suggested by the fact that both cracking and healing have been observed, the $K_{\mathrm{I}}$ throughout our experiments is not surprisingly distributed close to the Griffith load [31]. The healing is induced by the stress intensity lower than the fracture toughness. Therefore, by our method the Griffith load (the mode I fracture toughness) can be determined. Applying the 2D modulus $(E)$ directly measured by our AFM indentation experiments (Supplemental Material [27]), the mode I fracture toughness of $1 L \mathrm{ReS}_{2}$ is determined as $2.5 \pm 0.2 \mathrm{MPam}^{1 / 2}$. Moreover, close to the Griffith load, the dynamics of the cracking is governed by the lattice-trapping energy barriers [22,23], which means the thermal energy is required to activate the cracking events. The lattice trapping barrier heights are controlled by the crack stress intensity. When the lattice trapping barriers for healing are lower than the barriers for crack advance, healing will occur.

By our serial in situ STEM imaging, the electron beam effects can be either included or excluded in the in situ crack tests. Two types of crack dynamics have been observed. One type is the cracking event when the electron beam scanning is over or close to the crack tip zone, in this case a discontinuous scanning line will appear in the image and we can measure the cracking length (defined as one step) during $c a .10 \mathrm{~ms}(19 \mu \mathrm{s} \times 512$ pixels in one scanning line) [Fig. 2(c) and Fig. S16 [27] ]. On the other hand, we also found in some serial STEM snapshots, the discontinuous lines as above are totally absent, however the cracks still propagated a few unit cells known from the two successive STEM snapshots before and after the crack moves. In these cases, the crack move events should occur 
(a)

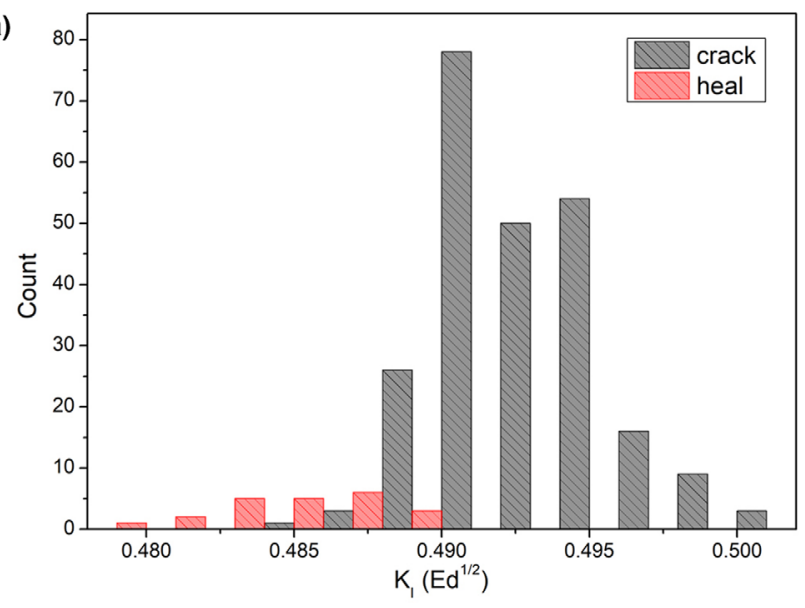

(b)

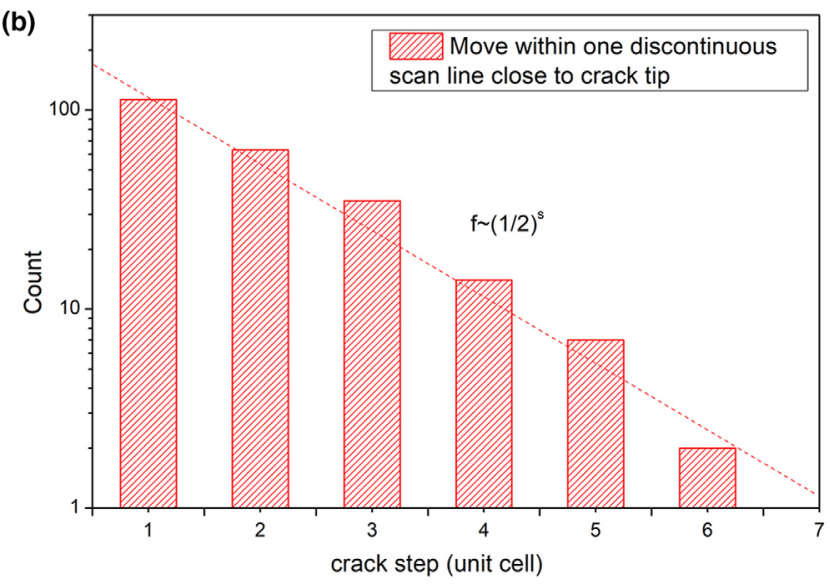

(c)

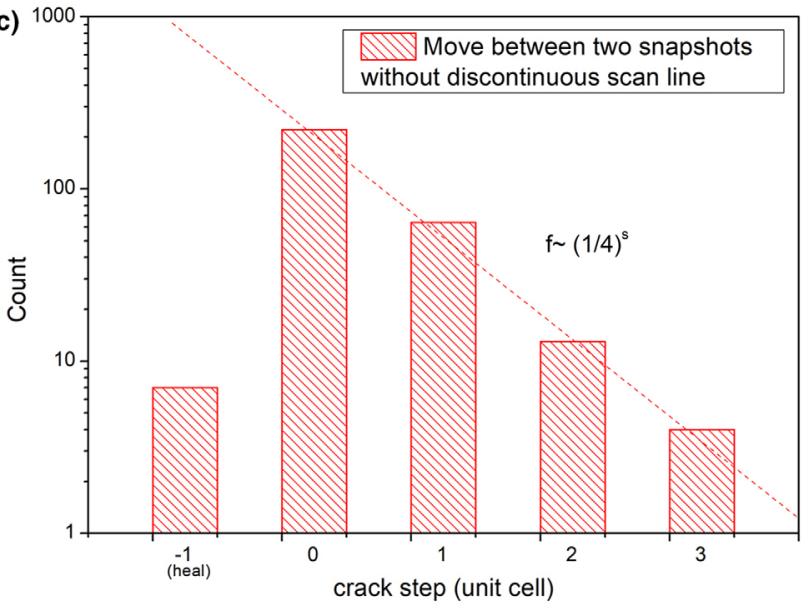

FIG. 4. The statistical results on the mode I crack and crack dynamics in $1 L \mathrm{ReS}_{2}$. (a) The histogram on the frequencies of loaded stress intensity $\left(K_{I}\right)$ for our experimental mode I crack moves, crack advance, and crack heal cases are distinguished. $E$ is modulus of and $d$ is the lattice spacing perpendicular to cracking $(y)$ direction for $\operatorname{ReS}_{2}$. (b) The histogram for frequencies of crack moving lengths (step) within one line scan of electron beam $(\sim 10 \mathrm{~ms})$, so it means the electron beam is close to the crack tip when crack moves. (c) The histogram for frequencies of crack moving lengths between two continuous snapshots of STEM images $(\sim 5 \mathrm{~s})$ when the electron beam is far away from the crack tip and no discontinuous lines are observed. when the electron beam is scanning over other areas on the sample with very small atomic displacements upon the crack moves, which are far from the crack tip zone ( $>5 \mathrm{~nm}$ distances to the tip), therefore the electron beam should have negligible effects on these crack moves. In this sense, by snapshots with or without discontinuous scanning lines, we can include or exclude the electron beam effects in our following dynamic analysis. The experimental statistical results on the observed frequencies $(f)$ versus crack move lengths $(s)$ for these two cases are presented in Figs. 4(b), 4(c), respectively.

In both cases, the frequencies have exponential decay on the crack move lengths, in agreement with the latticetrapped crack model. The crack propagation can be discretized down to individual moves (each single chemical bonding rupture) trapped by certain energy barriers. As the loading in our in situ experiments is controlled very close to Griffith load [Fig. 4(a)], the lattice trapping barriers can be considered as constant. Thus the frequencies of crack lengths should follow $f \sim z^{s}$ (see Supplemental Material [27] for details of modeling), where $z=$ $\nu(\tau / 6) \exp (\Delta E / k T), v$ is the vibration frequency and $\tau$ is the time interval for STEM snapshots. Using the experimentally obtained $z$ values in Fig. 4(c) and $v$ estimated by average phonon frequencies, the energy barrier for lattice trapping model without electron beam effect can be determined as $0.78 \mathrm{eV}$. As there are four times of bonding ruptures in one crack propagating over one unit cell in $1 L \mathrm{ReS}_{2}$, four sub-barrier heights in one unit cell obtained by the DFT simulation are between 0.4 to $0.8 \mathrm{eV}$ (Fig. S17 [27]). Since $0.8 \mathrm{eV}$ is quite close to our experimentally obtained $0.78 \mathrm{eV}$, our experiments here suggested the lattice trapping barrier should be mainly attributed to the highest sub-barrier.

Further, applying this barrier height for analyzing the data in Fig. 4(b), the effective temperature of the lattice affected by electron beam can be estimated as $374 \mathrm{~K}$, which means the effective lattice temperature of crack tip due to beam scanning right on the crack tip has been raised by around $76 \mathrm{~K}$ (a combined effect of knock-on and radiolysis). Using the heat dissipation model in two dimensions, the region close to the scanning beam (within $1 \mathrm{~nm}$ ) can experience an over fivefold temperature rise than the region far away from the beam $(>5 \mathrm{~nm})$, hence the beam effect on the crack tip when electron beam is out of the $5 \mathrm{~nm}$ crack tip zone is negligible (temperature rise less than $10 \mathrm{~K}$ ), and the intrinsic lattice-trapped barrier without irradiation damage (atomic sputtering or bonding dissociation) is confirmed.

Our in situ STEM observations have experimentally unveiled the atomic structures of propagating latticetrapped crack tips. We observed entirely brittle fracture down to the atomic scale without tip blunting in $2 \mathrm{D} \mathrm{ReS}_{2}$. The fracture toughness and lattice-trapping energies for cracks are measured by our direct atomic-scale imaging. We have experimentally demonstrated that, beyond the 
asymptotic field description in classical theories, the atomistic process in the singular crack tip zone is basically the sequential atomic bonding dissociation assisted by thermal energy. Since the lattice trapping barrier is highest for the Griffith load, and can be reduced by the increased loading or even totally vanish above the athermal loading, it is essential to maintain the experimental condition close to the Griffith load to achieve the high-resolution atomic scale imaging of the propagating crack tips. In short, our work has opened new avenues to explore the atomistic fracture mechanisms.

This work was supported by National Science Foundation of China (Projects No. 51872248, No. 21703076, No. 51922113), the Hong Kong Research Grant Council under Collaborative Research Fund (Project No. C602114EF), Early Career Scheme (Projects No. 25301018, No. 21303218), General Research Fund (Project No. 15302419), City University of Hong Kong (Project No. 9610387), Polytechnic Unviersity (ZVGH, ZVRP), Natural Science Foundation of Jiangsu Province of China (Project No. BK20170466), 333 High-level Talents Cultivating Project of Jiangsu Province (Project No. BRA2018341), Natural Science Research Program of Jiangsu Higher Education Institution (18KJA140001) and Shenzhen Science and Technology Innovation Commission (Project No. JCYJ20170818104717087). Fund of Key Laboratory of Advanced Materials of Ministry of Education. We thank Weibo Zhao for assistances in manuscript preparation.

"These authors contributed equally to this work.

†thuchly@ cityu.edu.hk

jiongzhao@polyu.edu.hk

[1] E. Bitzek, J. R. Kermode, and P. Gumbsch, Int. J. Fract. 191, 13 (2015).

[2] J. Cook and J. E. Gordon, Proc. R. Soc. Lond. A 282, 508 (1964).

[3] J. R. Rice and R. Thomson, Philos. Mag. A 29, 73 (1974).

[4] J. W. Hutchinson, J. Mech. Phys. Solids 16, 13 (1968).

[5] F. F. Abraham, D. Brodbeck, R. A. Rafey, and W. E. Rudge, Phys. Rev. Lett. 73, 272 (1994).

[6] J. A. Hauch, D. Holland, M. P. Marder, and H. L. Swinney, Phys. Rev. Lett. 82, 3823 (1999).

[7] Z. Zhang, A. Kutana, and B. I. Yakobson, Nanoscale 7, 2716 (2015).
[8] S. S. Terdalkar, S. Huang, H. Yuan, J. J. Rencis, T. Zhu, and S. Zhang, Chem. Phys. Lett. 494, 218 (2010).

[9] D. R. Clarke and K. T. Faber, J. Phys. Chem. Solids 48, 1115 (1987).

[10] B. R. Lawn, B. J. Hockey, and S. M. Wiederhorn, J. Mater. Sci. 15, 1207 (1980).

[11] C. Lee, X. Wei, J. W. Kysar, and J. Hone, Science 321, 385 (2008).

[12] P. Zhang, L. Ma, F. Fan, Z. Zeng, C. Peng, P. E. Loya et al., Nat. Commun. 5, 3782 (2014).

[13] T. H. Ly, J. Zhao, M. O. Cichocka, L. J. Li, and Y. H. Lee, Nat. Commun. 8, 14116 (2017).

[14] S. Wang, Z. Qin, G. S. Jung, F. J. Martin-Martinez, K. Zhang, M. J. Buehler, and J. H. Warner ACS Nano 10, 9831 (2016).

[15] B. R. Lawn, K. Jakus, and A. C. Gonzalez, J. Am. Ceram. Soc. 68, 25 (1985).

[16] S. Ito and M. Tomozawa, J. Am. Ceram. Soc. 65, 368 (1982).

[17] C. Cao, S. Mukherjee, J. Y. Howe, D. D. Perovic, Y. Sun, C. V. Singh, and T. Filleter, Sci. Adv. 4, eaao7202 (2018).

[18] S. Kondo, A. Ishihara, E. Tochigi, N. Shibata, and Y. Ikuhara, Nat. Commun. 10, 2112 (2019).

[19] J. G. Swadener, M. I. Baskes, and M. Nastasi, Phys. Rev. Lett. 89, 085503 (2002).

[20] D. Holland and M. Marder, Phys. Rev. Lett. 80, 746 (1998).

[21] H. Tan and W. Yang, Int. J. Fract. 77, 199 (1996).

[22] R. Thomson, C. Hsieh, and V. Rana. J. Appl. Phys. 42, 3154 (1971).

[23] T. Zhu, J. Li, and S. Yip, Proc. R. Soc. A 462, 1741 (2006).

[24] M. J. Buehler, H. Tang, A. C. T. van Duin, and W. A. Goddard III, Phys. Rev. Lett. 99, 165502 (2007).

[25] M. Chhowalla, H. S. Shin, G. Eda, L.-J. Li, K. P. Loh, and H. Zhang Nat. Chem. 5, 263 (2013).

[26] Y. C. Lin et al. ACS Nano 9, 11249 (2015).

[27] See Supplemental Material at http://link.aps.org/supplemental/ 10.1103/PhysRevLett.125.246102 for experimental details, notes and supplementary data.

[28] G. Kresse and J. Furthmuller, Phys. Rev. B 54, 11169 (1996).

[29] J. L. Rouviere and E. Sarigiannidou, Ultramicroscopy 106, 1 (2005).

[30] J. S. Ferenc and Z. Néda, Physica (Amsterdam) 385A, 518 (2007).

[31] A. A. Griffith VI., Phil. Trans. R. Soc. A 221, 163 (1921).

[32] G. R. Irwin, Proc. 7th Sagamore Res. Conf. (1961), Vol. 4, p. 63.

[33] J. R. Rice, J. Appl. Mech. 35, 379 (1968). 